\title{
LAESI mass spectrometry imaging as a tool to differentiate the root metabolome of native and range-expanding plant species
}

\author{
Purva Kulkarni $^{1}$ (D) $\cdot$ Rutger A. Wilschut $^{1} \cdot$ Koen J. F. Verhoeven ${ }^{1} \cdot$ Wim H. van der Putten $^{1,2} \cdot$ Paolina Garbeva $^{1}$
}

Received: 5 June 2018 / Accepted: 14 August 2018 / Published online: 23 August 2018

(c) The Author(s) 2018

\begin{abstract}
Main conclusion LAESI-MSI, an innovative high-throughput technique holds a unique potential for untargeted detection, profiling and spatial localization of metabolites from intact plant samples without need for extraction or extensive sample preparation.
\end{abstract}

Our understanding of chemical diversity in biological samples has greatly improved through recent advances in mass spectrometry (MS). MS-based-imaging (MSI) techniques have further enhanced this by providing spatial information on the distribution of metabolites and their relative abundance. This study aims to employ laser-ablation electrospray ionization (LAESI) MSI as a tool to profile and compare the root metabolome of two pairs of native and range-expanding plant species. It has been proposed that successful range-expanding plant species, like introduced exotic invaders, have a novel, or a more diverse secondary chemistry. Although some tests have been made using aboveground plant materials, tests using root materials are rare. We tested the hypothesis that range-expanding plants possess more diverse root chemistries than native plant species. To examine the root chemistry of the selected plant species, LAESI-MSI was performed in positive ion mode and data were acquired in a mass range of m/z 50-1200 with a spatial resolution of $100 \mu \mathrm{m}$. The acquired data were analyzed using in-house scripts, and differences in the spatial profiles were studied for discriminatory mass features. The results revealed clear differences in the metabolite profiles amongst and within both pairs of congeneric plant species, in the form of distinct metabolic fingerprints. The use of ambient conditions and the fact that no sample preparation was required, established LAESI-MSI as an ideal technique for untargeted metabolomics and for direct correlation of the acquired data to the underlying metabolomic complexity present in intact plant samples.

Keywords Mass spectrometry imaging · Ambient imaging $\cdot$ Comparative metabolomics $\cdot$ Plant root $\cdot$ Metabolic profiling · Range expansion

\begin{tabular}{|c|c|c|c|}
\hline \multicolumn{2}{|c|}{ Abbreviations } & CJ & Centaurea jacea $\mathrm{L}$ \\
\hline MS & Mass spectrometry & CS & Centaurea stoebe $\mathrm{L}$ \\
\hline Mass spectrometry imaging & Mass spectrometry imaging & GM & Geranium molle L \\
\hline \multicolumn{2}{|r|}{ Laser-ablation electrospray ionization } & GP & Geranium pyrenaicum Burm \\
\hline & & $m / z$ & Mass by charge \\
\hline \multirow{3}{*}{\multicolumn{2}{|c|}{$\begin{array}{l}\text { Electronic supplementary material The online version of this } \\
\text { article (https://doi.org/10.1007/s00425-018-2989-4) contains } \\
\text { supplementary material, which is available to authorized users. }\end{array}$}} & GC & Gas chromatography \\
\hline & & TOF & Time-of-flight \\
\hline & & PCA & Principal component analysis \\
\hline \multicolumn{2}{|c|}{$\begin{array}{l}\triangle \text { Purva Kulkarni } \\
\text { P.Kulkarni@ nioo.knaw.nl }\end{array}$} & $\mathrm{FC}$ & Fold change \\
\hline \multicolumn{2}{|r|}{$\begin{array}{l}\text { Netherlands Institute of Ecology (NIOO-KNAW), } \\
\text { Droevendaalsesteeg 10, 6708 PB Wageningen, } \\
\text { The Netherlands }\end{array}$} & & \\
\hline \multicolumn{2}{|r|}{$\begin{array}{l}\text { Laboratory of Nematology, Wageningen University } \\
\text { and Research Centre, PO Box 8123, 6700 ES Wageningen, } \\
\text { The Netherlands }\end{array}$} & & \\
\hline
\end{tabular}




\section{Introduction}

Detection of plant metabolites is extremely challenging, as there is no single-instrument platform available to effectively measure their overall coverage. During the last decade, mass spectrometry imaging (MSI) has emerged as a valuable tool, with numerous applications in the field of biological sciences. This analytical technique enables label-free, highresolution spatial mapping of a large variety of biomolecules along with providing qualitative and quantitative chemical information, in a single experiment (Petras et al. 2017). Identical to traditional mass spectrometry, during MSI it is important to ionize the sample to form ions suitable for mass analysis. Different ionization methods exist for MSI; however, many of them require artificially altering the native biochemical status of the system under study, for example, by the application of a matrix, and are mainly operated under vacuum. Recently developed ambient ionization approaches such as laser-ablation electrospray ionization (LAESI) allow direct analysis of biological samples in a matrix-free, native atmospheric condition with minimal to no sample preparation, in a significantly shorter analysis time (Cooks et al. 2006; Wu et al. 2013). This opens up possibilities for in situ chemical analysis in biological systems.

LAESI-MSI is particularly tailored for biological samples that are rich in water content (Nemes and Vertes 2007). In this technique, the sample under investigation is mounted on a sample stage and is ablated using a focused mid-infrared laser pulse, under atmospheric conditions. This ablation ejects a mixture of molecules, clusters, and particulate matter in microscopic volumes from the sample, in the form of a plume. The catapulted biomolecules then coalesce with charged droplets, produced by an electrospray to become ionized (Chen et al. 2006; Chen and Vertes 2008). MSI using the LAESI ionization approach is realized by rastering the sample surface at pre-defined coordinates with a laser beam, where at each coordinate position the generated ions pass through the mass analyzer and a mass spectrum is recorded. LAESI-MSI has shown considerable success in revealing the lateral and cross-sectional distribution of primary and secondary metabolites for a range of plant-related samples, along with providing chemical information from deeper parts of the tissue section (Bjarnholt et al. 2014). LAESI equipped with a sharpened optical fiber tip has also been widely used to perform in situ metabolic profiling of single cells from plant and animal samples (Shrestha and Vertes 2009).

Here, we aim to demonstrate the potential of LAESI-MSI as an analytical technique for the direct metabolite profiling of plant samples. We applied LAESI-MSI in a comparative metabolic profiling study on two pairs of non-native, rangeexpanding plant species and congeneric native plant species.
In response to recent climate warming, many plant species have expanded their range to higher latitudes and altitudes (Walther et al. 2002; Le Roux and McGeoch 2008). It is thought that plant secondary chemistry is an important factor determining the invasive success of exotic plant species. The novel chemistry of invasive exotic plant species may effectively control defenses against insect herbivores and other natural enemies (Cappuccino and Arnason 2006). Such 'novel chemistry' has been shown to potentially suppress native plant species directly through allelopathy (Callaway and Aschehoug 2000) or indirectly through the suppression of the fungal mutualists of native plant species (Marler et al. 1999; Stinson et al. 2006). Moreover, due to this difference in chemistry, native generalist herbivores may perform less well on exotic plant species than on related native plant species (Schaffner et al. 2011), potentially leading to a reduced herbivore pressure on exotics compared to natives. The poor performance of generalist herbivores has also been linked to the high diversity of metabolites produced by exotic plant species compared to native plant species (Macel et al. 2014). This suggests that chemically diverse plant species may be prone to become abundant when they are introduced in a new area where the local herbivores are poorly adapted to neutralize, or circumvent the novel defenses.

In this study, we use LAESI-MSI as a high-throughput tool for untargeted comparative metabolomics of intact plant roots of native and range-expanding plant species. For this, we use two range-expanding plant species that are currently expanding in northwestern Europe, Centaurea stoebe L. and Geranium pyrenaicum Burm. f., and their respective congeneric native species Centaurea jacea L. and Geranium molle L. With this study, we demonstrate the suitability of LAESI-MSI for untargeted metabolomics profiling and we give insights in the potential chemical novelty of rangeexpanding plant species in comparison to congeneric-related native plant species.

\section{Materials and methods}

\section{Plant species and root collection}

The seeds used for all four plant species originated from natural populations in natural areas in The Netherlands, where the range expanders are immigrating. Seeds of $G$. molle and $C$. stoebe were collected directly from the field. For $C$. jacea, seeds were collected from plants growing in an experimental garden, whereas the mother plants were germinated from field-collected seeds. Seed production company Cruydt-hoeck (Groningen, The Netherlands), that grows plants originating from field-collected seeds, delivered the seeds for $G$. pyrenaicum. For all plant species, the seeds were surface-sterilized by washing for $3 \mathrm{~min}$ in a $10 \%$ bleach 
solution, followed by rinsing with demineralized water, after which they were germinated on glass beads. After 20 days, the seedlings were collected for LAESI analysis.

\section{LAESI mass spectrometry imaging}

The LAESI-MSI of intact roots collected from the seedlings was carried out on a Protea Biosciences DP-1000 LAESI system (Protea Bioscience Inc., Morgantown) coupled to a Waters model Synapt G2S (Waters Corporation) mass spectrometer. The LAESI system was equipped with a 2940-nm mid-infrared laser yielding a spot size of $100 \mu \mathrm{m}$. The laser was set to fire ten times per $x-y$ location (spot) at a frequency of $10 \mathrm{~Hz}$ and $100 \%$ output energy. The system was set to shoot at 105 locations per plant root (grid of $21 \times 5$ positions). A syringe pump was delivering the solvent mixture of methanol/water/formic acid (50:50:0.1\% v/v) at 2 $\mu \mathrm{L} / \mathrm{min}$ to a PicoTip $(5 \mathrm{~cm} \times 100 \mu \mathrm{m}$ diameter $)$ stainless steel nanospray emitter operating in positive ion mode at 3800 V. The LAESI was operated using LAESI Desktop Software V2.0.1.3 (Protea Biosciences Inc.). The Time of Flight (TOF) mass analyzer of the Synapt G2S was operated in V-reflectron mode at a mass resolution of 18.000-20.000. The source temperature was $150{ }^{\circ} \mathrm{C}$, and the sampling cone voltage was $30 \mathrm{~V}$. The data were acquired in a mass range of $m / z$ 50-1200. The acquired MS data were lock mass corrected post-data acquisition using leucine encephalin $\left(\mathrm{C}_{28} \mathrm{H}_{37} \mathrm{~N}_{5} \mathrm{O}_{7}, m / z=556.2771\right)$, which was added in the spray as an internal standard.

\section{Data processing, peak detection and chemometrics}

All the acquired Waters .raw data files were first pre-processed to remove noise and to make the data comparable. Since the root samples used in this study were tiny, many LAESI ablation spots constituted the background on which the root samples were placed. To avoid including the mass spectra purely consisting of spectral signals from the background, 50 ablation spots per sample replicate, present on the root section were selected manually. The selected ablation spots for every sample replicate are displayed in Online Resource 1 as Supplementary Fig. 1. The mass spectra arising from the spots colored in green are included in the study whereas those in red have been excluded.

The spectra from all the 50 selected spots for each replicate were averaged. Processing of these mass spectra involved multiple steps. An overview of the data processing steps applied is provided in Fig. 1. First, square root transformation was applied to the data to stabilize the variance. Then, baseline correction was performed to enhance the contrast of peaks to the baseline. For better comparison of intensity values and to remove small batch effects, Total-IonCurrent (TIC)-based normalization was applied. This was followed by spectral alignment and peak detection to extract a list of significant mass features for each sample replicate. In the end, a mass feature matrix was generated with sample replicates in columns and mass features in rows. This feature matrix was used to perform chemometric analysis. The preprocessing and peak-detection steps were applied using $\mathrm{R}$ scripts developed in-house and the functions available within the MALDIquant R package (Gibb and Strimmer 2012).

To perform multivariate analysis, the feature matrix was imported into Metaboanalyst 3.0 (Xia et al. 2015). Principal

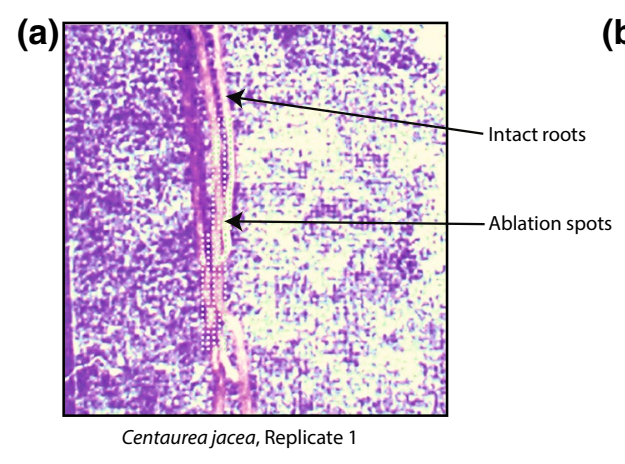

(d)

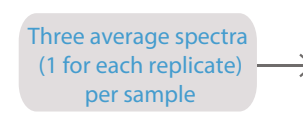

Fig. 1 Data preparation and processing steps applied post-acquisition. a Optical image for the intact roots of a single replicate of $C$. jacea with labeled ablation spots. b Ablation spots present on the root
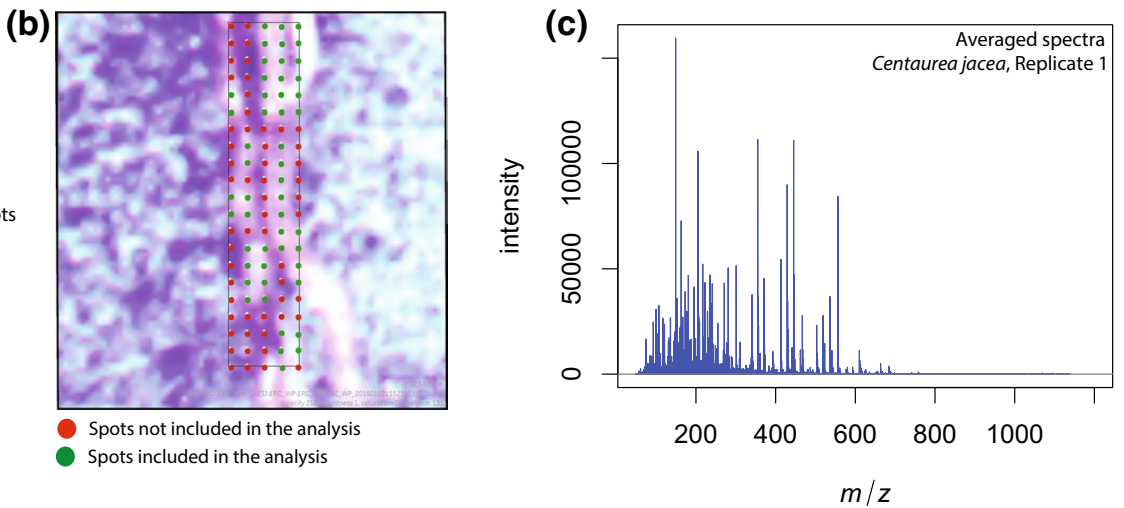
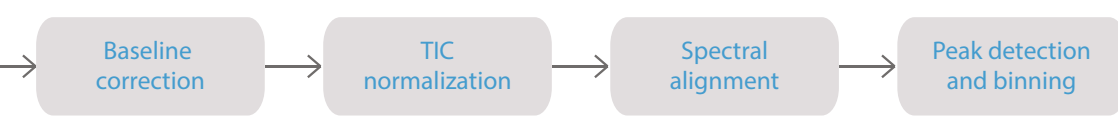

selected (in green) for further analysis. c Averaged spectra acquired from all the 50 selected spots per replicate. $\mathbf{d}$ Data pre-processing and peak-detection steps applied to all spectra for a sample 
component analysis (PCA) was initially applied to visualize the intrinsic spectral differences in the non-native, rangeexpanding plant species and congeneric native plant species. To get an overview of the differences amongst the samples, a dendrogram showing clustering of the sample replicates was generated using the Euclidean distance measure and the Ward's clustering algorithm. To visualize the number of differential metabolites in non-native, range-expanding plant species and congeneric native plant species, a pairwise comparative analysis was performed. To graphically illustrate these differences volcano plots were generated. Metabolites with a fold change (FC) threshold of 2 on the $x$ axis and a $t$ tests threshold ( $p$ value) of 0.1 on the $y$ axis were considered significant. Box plots for selected significant metabolites were created to display changes in the concentration of native and range-expanding species. Corresponding accurate ion intensity maps $( \pm 1 \mathrm{ppm})$ displaying spatial distribution for these selected mass features were created using the ProteaPlot software V2.0.1.3 (Protea Biosciences Inc., Morgantown, WV). The intensity values for the selected ion maps were normalized to the maximum intensity within the image, measured for each mass value individually. Venn diagrams were drawn using the jvenn tool (Bardou et al. 2014) to plot the number of shared and unique metabolites for each pair of samples.

\section{Results and discussion}

\section{Untargeted metabolite profiling and multivariate analysis}

Untargeted metabolomic studies are exploratory in nature and usually result in extremely large and multi-dimensional datasets. Analyses of such datasets using chemometric tools can hugely aid data interpretation.

The representative averaged pre-processed spectra for each replicate belonging to the different plant species exhibit some visual distinction in mass spectra between the two plant genera (Fig. 2). This distinction between Centaurea and Geranium samples was further confirmed by unsupervised hierarchical clustering of the mass feature matrix (Fig. 3a). Within the two genera, the different plant species were mostly clearly separated based on their chemical
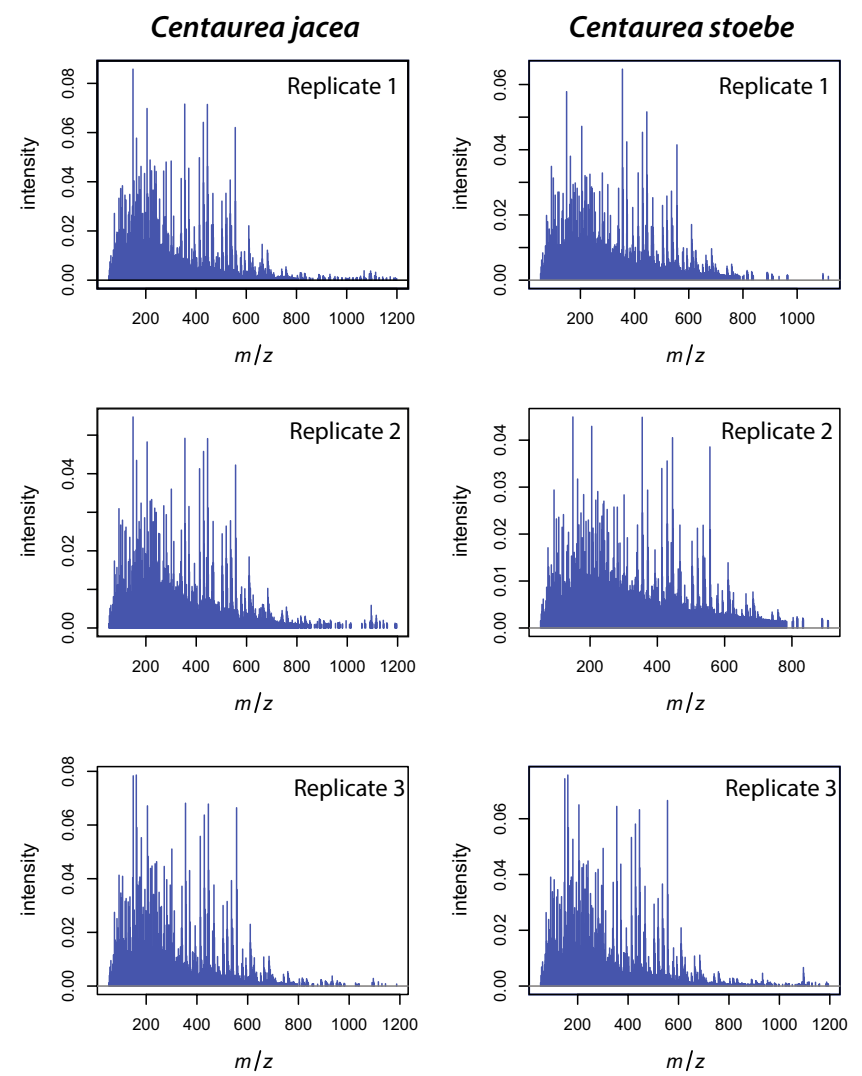

Fig. 2 Metabolic profiling and comparison of LAESI-MS spectra from native and range-expanding plant species. Each representative mass spectra is generated by averaging and pre-processing the signals acquired in positive ion mode, arising from the 50 ablation spots pre-
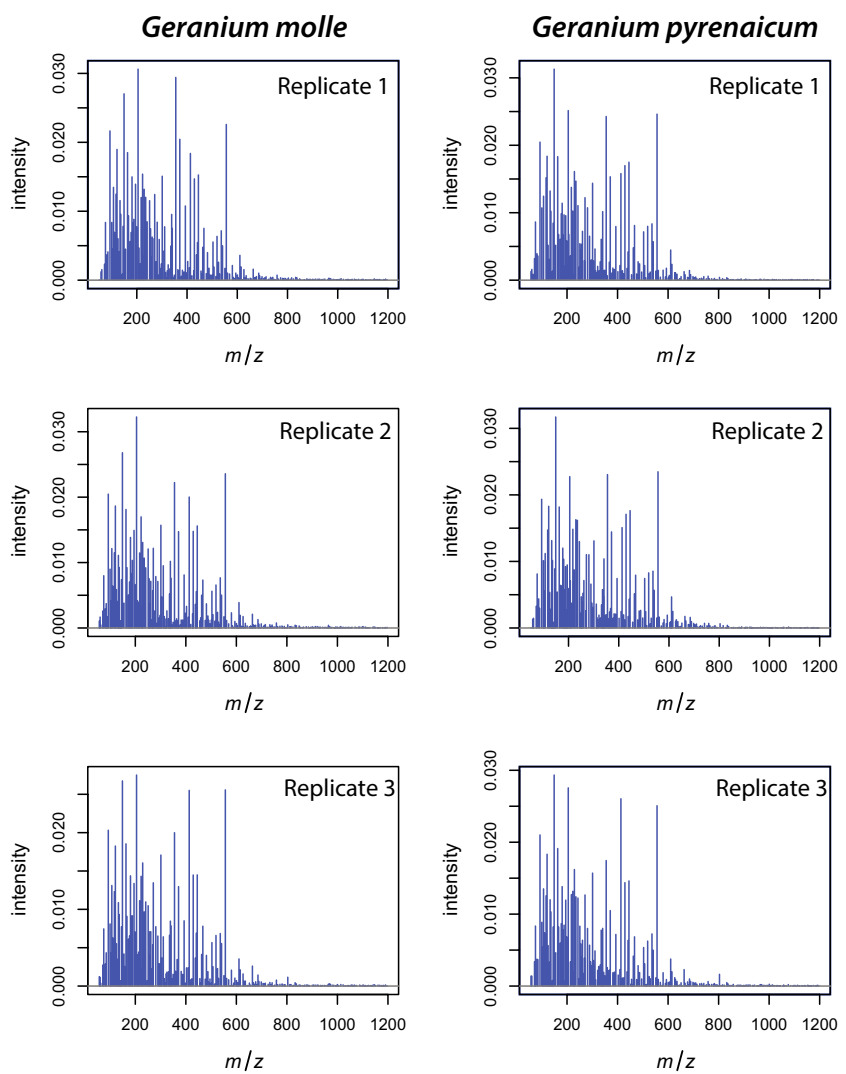

sent on the imaged root sample for each replicate. The averaged preprocessed mass spectra are displayed for the three replicates of native species (C. jacea and $G$. molle) and the three replicates for rangeexpanding plant species (C. stoebe L. and G. pyrenaicum) 
(a)

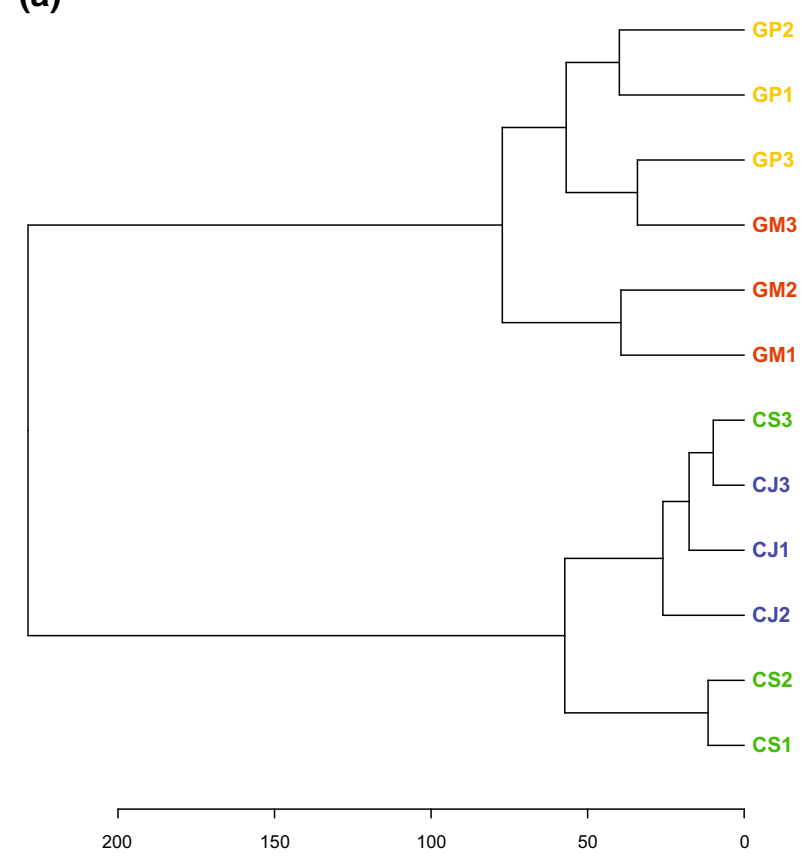

Fig. 3 Dendrogram and Principal component analysis (PCA) score plot for the selected native and range-expanding species. a Species clustering represented as a dendrogram (distance measure used is Euclidean and clustering algorithm is ward). Each node in the dendrogram corresponds to a single replicate belonging either to the

Table 1 Overview of the number of metabolites detected in each sample replicate after pre-processing and peak detection of the acquired LAESI-MSI datasets

\begin{tabular}{lllll}
\hline Replicate & C. jacea (CJ) & C. stoebe (CS) & G. molle (GM) & $\begin{array}{l}\text { G. pyr- } \\
\text { enaicum } \\
\text { (GP) }\end{array}$ \\
\hline 1 & 283 & 332 & 143 & 129 \\
2 & 204 & 301 & 151 & 127 \\
3 & 286 & 286 & 131 & 122 \\
\hline
\end{tabular}

features, with the exception of one of the $C$. stoebe replicates (Fig. 3a).

Visual comparison of the representative mass spectrum for each sample group can be used to broadly study the differing metabolic profiles. To further examine these differences and similarities between the root metabolic profiles of the four plant species, we employed PCA. The first two selected principal component axes explain over $75 \%$ of cumulative variance amongst the samples (Fig. 3b). Samples from different plant genera were strongly separated along the first PC-axis ( 57\%), whereas the separation along the second PC-axis $(\sim 18 \%)$ corresponded with within-genus variation (Fig. 3b). Together with hierarchical clustering (Fig. 3a), these results indicate a strong phylogenetic signal (b)

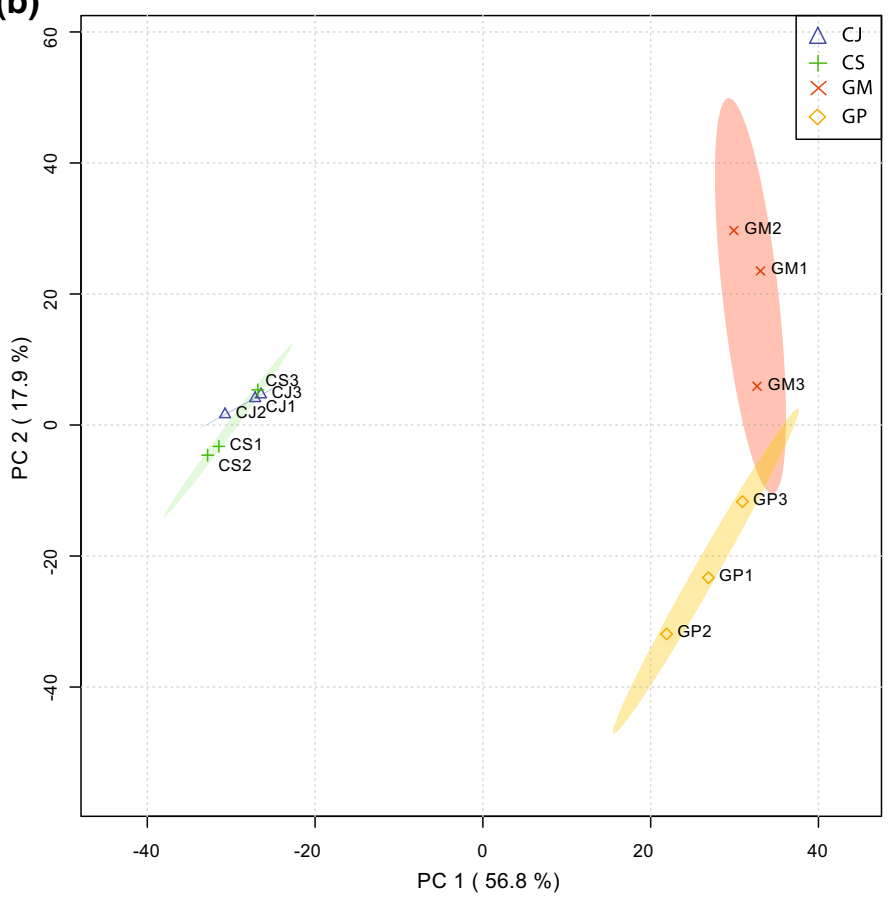

range-expanding or to the congeneric native plant species. b The PCA score plot displays the total explained variance of $>70 \%$ for component 1 and component 2. Ovals represent $95 \%$ confidence intervals. Each oval represents a sample group and each point represents a single sample

in root chemistry, as between-genus variation is considerably stronger than within-genus variation (Senior et al. 2016).

The number of mass features detected for each LAESIMSI acquisition after performing data pre-processing and peak detection clearly shows that there are more mass features detected for the replicates of Centaurea as compared to those of Geranium (Table 1). The two Centaurea species shared 314 metabolites, whereas 53 metabolites were unique to either one of the species (Fig. 4a). Interestingly, 49 of these metabolites were unique for range-expanding $C$. stoebe, whereas only four were unique for native $C$. jacea. In contrast, for native $G$. molle more unique metabolites were detected than in range-expanding G. pyrenaicum (Fig. 4b). These results are in line with a previous study in which only root volatiles were examined (Wilschut et al. 2017) and indicate that range-expanding plants do not necessarily possess a more unique root chemistry than related natives.

To visualize the statistically significant metabolites for the two Centaurea species, a volcano plot was constructed (Fig. 5a). As seen in Fig. 5a, in total 367 metabolites were detected in genus Centaurea. Within this, ten mass features (shown in green) that are located in upper right quadrant of the plot, indicate that their concentration is significantly higher in native species $C$. jacea than in range-expanding species $C$. stoebe. The five mass features (shown in red) 


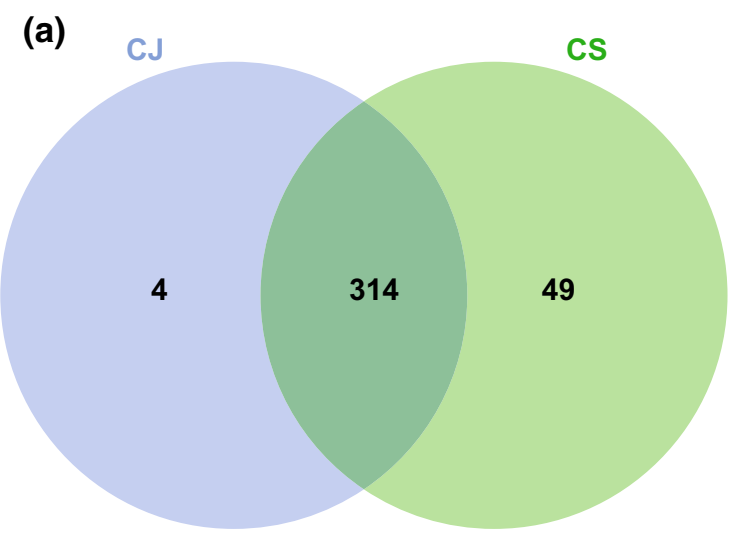

Fig. 4 Venn diagram showing overlapping and unique metabolites associated with native and range-expanding plant species. a Venn diagram for $C$. jacea $(\mathrm{CJ})$ and $C$. stoebe $(\mathrm{CS})$. b Venn diagram for $G$.

that are observed in the upper left quadrant indicate that their concentration is significantly lower in native species $C$. jacea than in range-expanding species $C$. stoebe. To examine the differences in metabolite concentrations for the $C$. jacea and $C$. stoebe pair, box-and-whisker plots were realized for four statistically significant metabolites chosen based on the volcano plot (Fig. 5b). The box-and-whisker plots and the ion intensity maps reveal that $\mathrm{m} / \mathrm{z} 84.9607, \mathrm{~m} / \mathrm{z} 159.0520$ and $\mathrm{m} / \mathrm{z} 557.290$ are highly abundant in native species $C$. jacea, whereas $\mathrm{m} / \mathrm{z} 272.9550$ are highly abundant in rangeexpanding species $C$. stoebe. Additionally, the corresponding ion intensity maps for these metabolites were also generated to visualize the changes on the spatial level in the imaged roots. The ion intensity maps can be seen alongside the box-and-whisker plots in Fig. 5b. Each ion map is plotted on the same color scale (depicted below the ion maps) ranging from 0 (blue meaning least intense) to 1 (red meaning most intense), to allow comparison of relative ion intensity between images.

Similar analysis was performed for the two Geranium species (Fig. 5c). For this pair, in total 175 metabolites were detected. Within these, 15 mass features (shown in green) that are located in the upper right quadrant of the plot, which indicates that their concentrations are significantly higher in native species $G$. molle than in range-expanding species G. pyrenaicum. The four mass features (shown in red) that are observed in the upper left quadrant indicate that their concentration is significantly lower in native species G. molle than in range-expanding species G. pyrenaicum. The box-and-whisker plots for the four statistically significant metabolites selected from the volcano plot for the pair G. molle and G. pyrenaicum are shown in Fig. 5d. The ion intensity maps for these statistically significant metabolites are shown alongside box-and-whisker plots. As it can be seen, $\mathrm{m} / z, 158.2647$ and $\mathrm{m} / \mathrm{z} 250.8271$ show high abundance

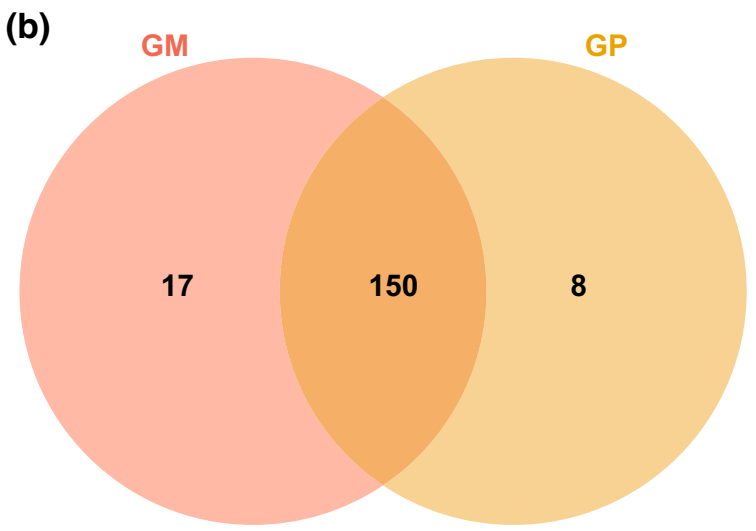

molle (GM) and G. pyrenaicum (GP). To construct the Venn diagram, a single mass feature was considered even if it was present in only one replicate for a specific sample species

Fig. 5 Volcano plots and box plots to demonstrate metabolite concentration differences observed in native and range-expanding plant species. a Volcano plot for $C$. jacea (CJ) vs. C. stoebe (CS). b Volcano plot for G. molle (GM) vs. G. pyrenaicum (GP). Each point in the volcano plot represents one metabolite. Significant metabolites were calculated with a fold change (FC) threshold of 2 on the $x$ axis and a $t$ tests threshold of 0.1 on the $y$ axis. The red and the green dots indicate statistically significant metabolites, and the gray dots below the FC threshold line represent statistically non-significant metabolites. The vertical FC threshold lines indicate an increase or decrease in concentration of metabolites. Negative $\log 2$ (FC) values indicated in red represent lower concentrations in native than in range-expanding species; positive values indicated in green represent higher concentrations of metabolites in native than in range-expanding species. The box plots for the detected metabolites and their corresponding ion intensity maps below each volcano plot display the localization of the selected metabolites that are significantly different in the respective native and range-expanding species. The signal intensity in the ion intensity maps is represented in rainbow color scale, in a mass window of $\pm 1 \mathrm{mDa}$

in native species G. molle, whereas $\mathrm{m} / \mathrm{z} 172.3829$ and $\mathrm{m} / \mathrm{z}$ 196.5855 display high abundance in range-expanding species G. pyrenaicum. All significant metabolites detected for Centaurea and Geranium samples are listed in Online Resource 1 as Supplementary Table 1.

Taken together, we demonstrated the utility of the unique ambient ionization ability of LAESI coupled with MSI as a high-throughput method to explore the chemical differences in the root metabolome between two pairs of native and range-expanding plant species. This technology provided an in situ analysis method capable of revealing differentially produced metabolites linked to each group. We detected clear differences in root chemical profiles within both pairs of range-expanding plant species and congeneric natives using untargeted LAESI-MSI approach. Interestingly, the range-expanding plant species Centaurea stoebe showed a 
(a) CJ and CS

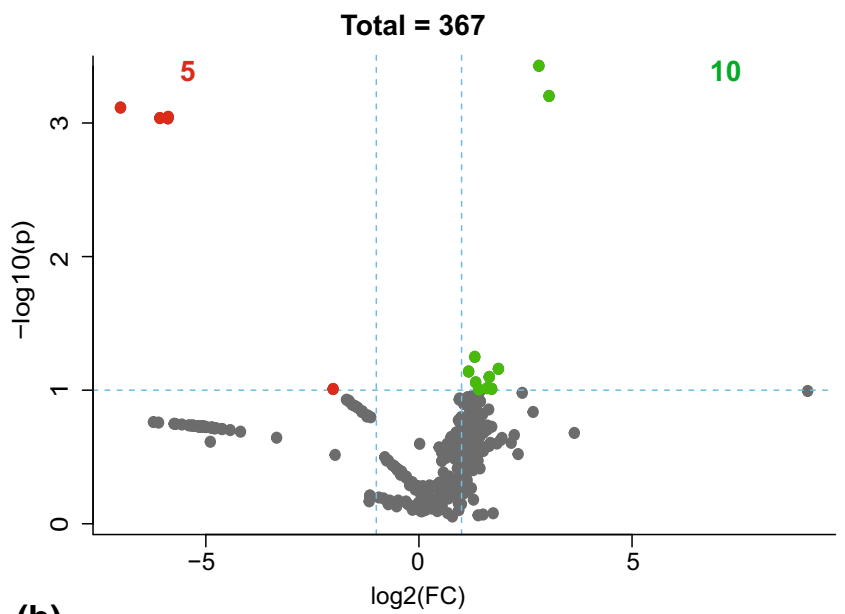

(b)

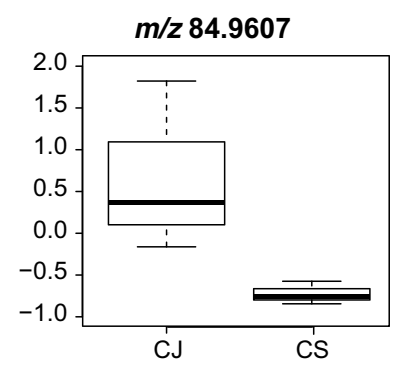

CJ
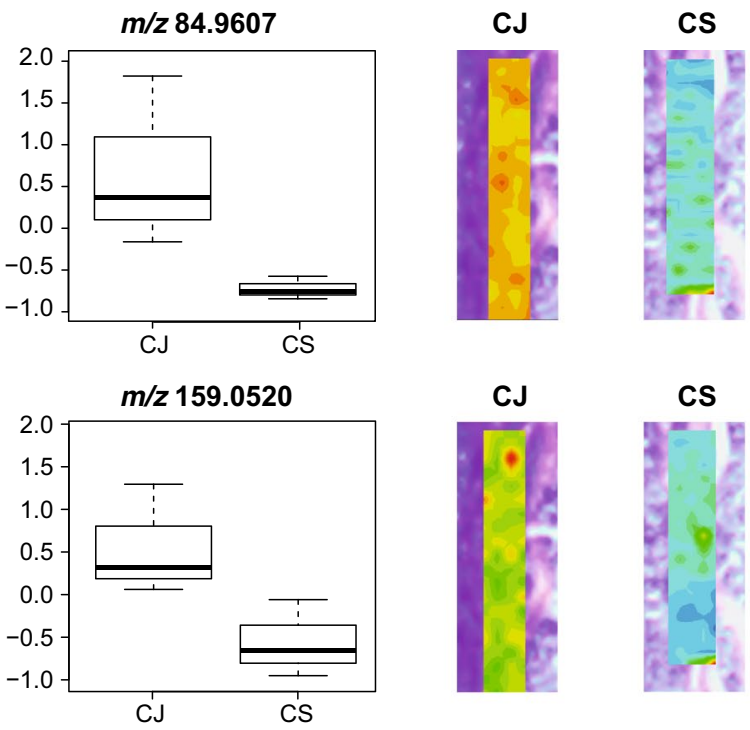

CJ
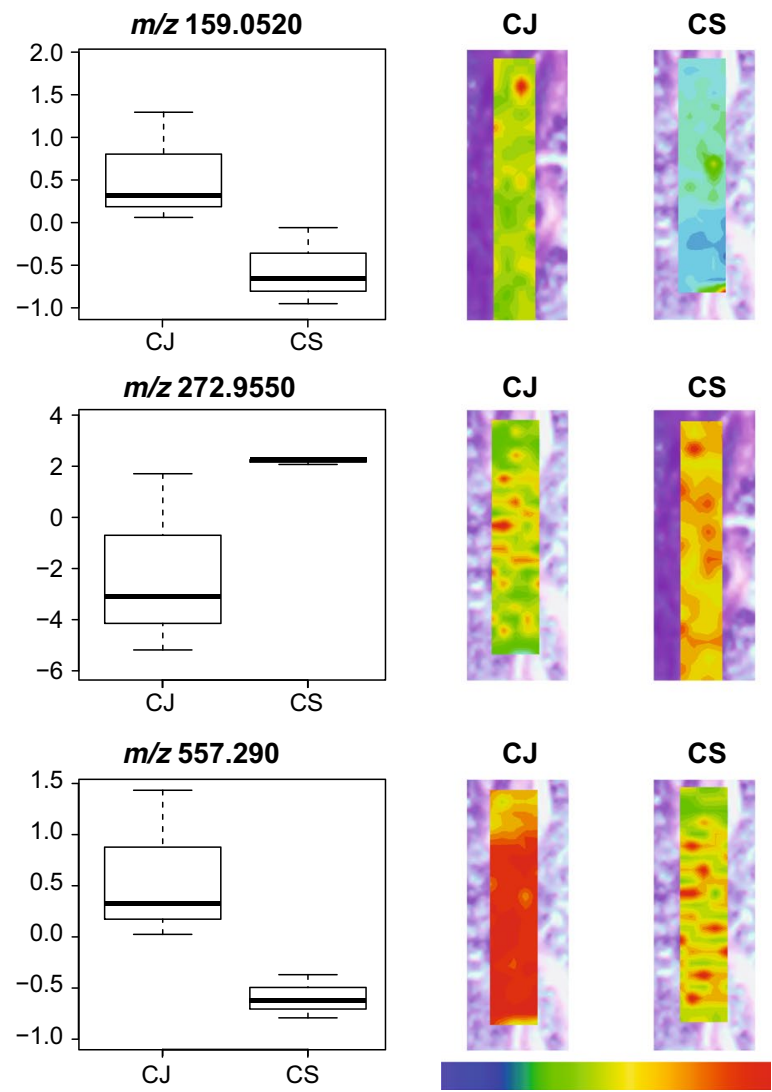

(c) GM and GP

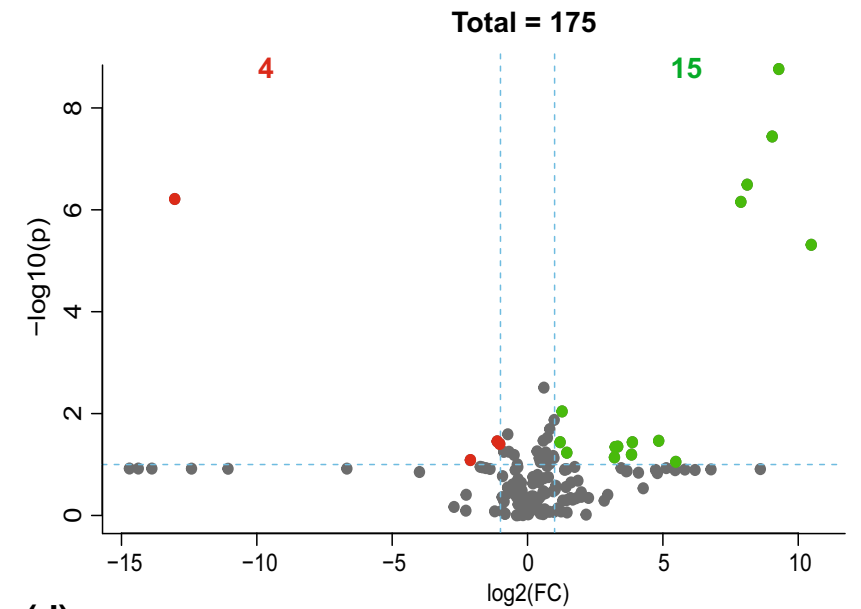

(d)
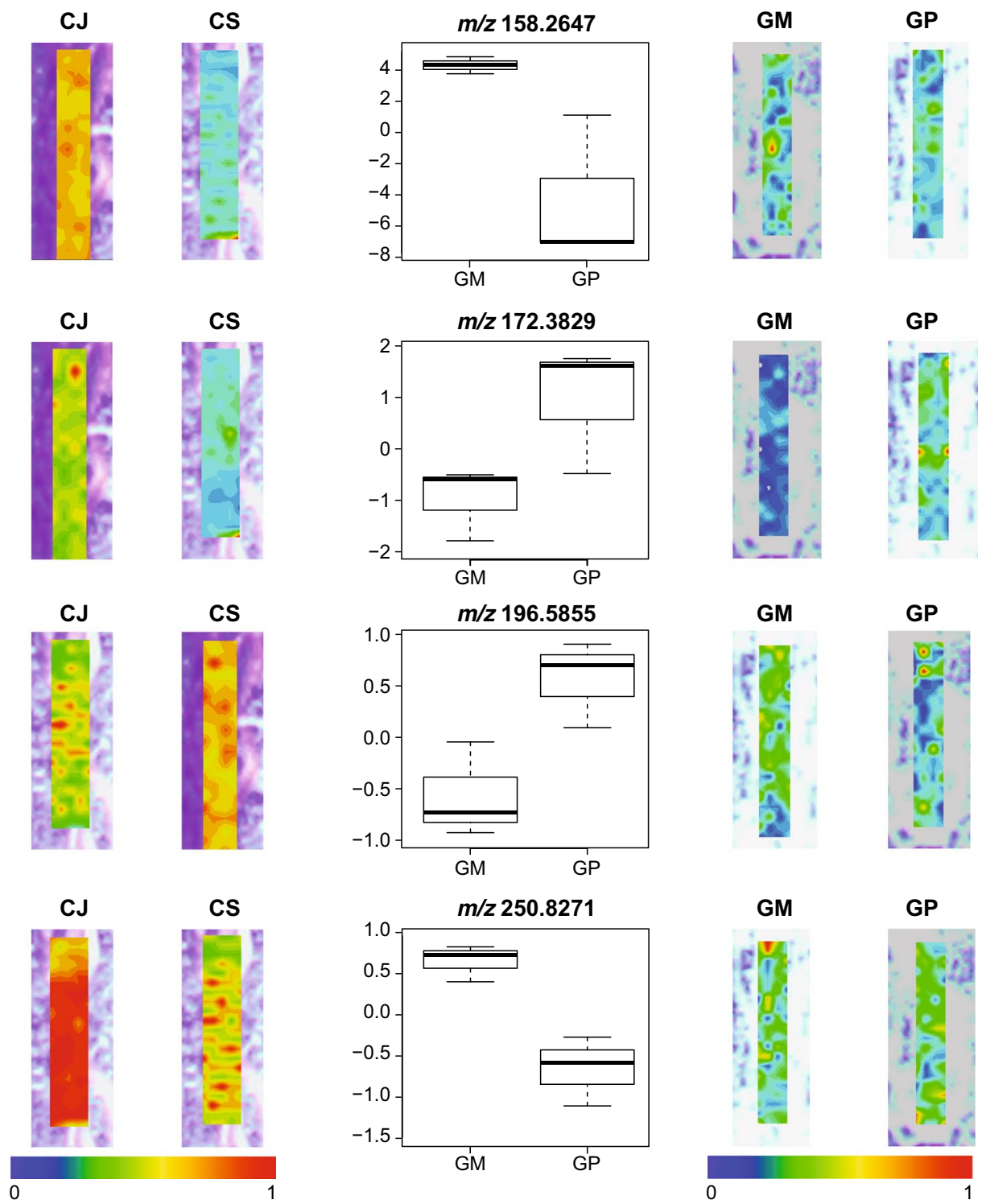

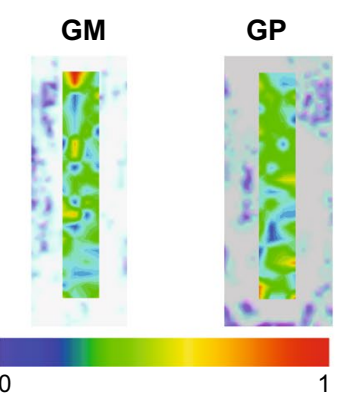


strongly unique root chemistry, which also may have enabled this species to become invasive in its introduced range in North America (Callaway and Ridenour 2004; Schaffner et al. 2011).

Furthermore, we demonstrated that LAESI-MSI can help to spatially elucidate the metabolite composition of the intact roots with minimal to no sample preparation. Our demonstration did not involve an exhaustive region-specific spatial analysis of the roots, but rather a 'proof-of-concept' by lateral profiling of the root samples. This allowed us to establish that LAESI-MSI of whole-root sections could reveal information on location-specific metabolite distribution without the need for any sample preparation. These results can help to reveal the role of single metabolites based on their location within the roots. The statistically significant biomolecules in the processed LAESI mass spectra can be putatively annotated by matching the observed masses with those of known metabolites using database searches. However, for confident compound identification it is best to couple LAESI mass spectrometry imaging with ion mobility separation (Stopka et al. 2017), which allows separation of isobaric species. Apart from this, LAESI-MSI with its feature of spatial mapping can hugely complement conventional extraction-based untargeted hyphenated-MS techniques like LC-MS or GC-MS. This can be further assisted by the application of tandem mass spectrometry for increased selectivity and structure assignments.

Overall, our results illustrate the feasibility of LAESI-MSI as a high-throughput technique for the detection and localization of metabolites from intact plant samples and gaining spatial information without the need for extensive sample preparation. The potential applications of this work could lead to rapid phenotyping of plant tissues as well as comparative untargeted metabolomics of different plant parts, a topic of considerable recent interest for plant research.

Author contribution statement PG, KJFV and RAW devised the project. PG and RAW oversaw the sample collection and the data acquisition. PK planned and performed the bioinformatics analysis, interpretation of results and prepared the figures. PK, RAW and PG wrote the manuscript. WHvdP, PG, KJFV and RAW provided their comments and contributed to substantial revision of the manuscript.

Acknowledgements We thank Frank Claassen from the department of Agrotechnology and Food Sciences at the Wageningen University for assistance with LAESI-MSI measurement and Julio Pereira da Silva for help with the experimental preparation. Purva Kulkarni is supported by the strategic project fund from the Netherlands Institute of Ecology (NIOO-KNAW). Rutger A. Wilschut and Wim H. van der Putten are supported by the ERC advanced grant ERC-Adv 26055290.
This is publication 6563 of the Netherlands Institute of Ecology (NIOO-KNAW).

\section{Compliance with ethical standards}

Conflict of interest The authors declare that they have no competing interests.

Availability of source code and requirements Project name: LAESIMSI-Root-Metabolomics. Project home page: https://github.com/ purvakulkarni7/LAESI-MSI-Root-Metabolomics (DOI: https://doi. org/10.5281/zenodo.1240843). Operating system(s): platform independent. Programming language: R. Other requirements: $R(\geq 3.2 .0)$, MALDIquant package, MALDIquantForeign package. License: GNU General Public License version 2.0 (GPLv2). Any restrictions to use by non-academics: none.

Open Access This article is distributed under the terms of the Creative Commons Attribution 4.0 International License (http://creativeco mmons.org/licenses/by/4.0/), which permits unrestricted use, distribution, and reproduction in any medium, provided you give appropriate credit to the original author(s) and the source, provide a link to the Creative Commons license, and indicate if changes were made.

\section{References}

Bardou P, Mariette J, Escudié F et al (2014) jvenn: an interactive Venn diagram viewer. BMC Bioinform 15:293. https://doi. org/10.1186/1471-2105-15-293

Bjarnholt N, Li B, D’Alvise J, Janfelt C (2014) Mass spectrometry imaging of plant metabolites-principles and possibilities. Nat Prod Rep 31:818-837. https://doi.org/10.1039/c3np70100j

Callaway RM, Aschehoug ET (2000) Invasive plants versus their new and old neighbors: a mechanism for exotic invasion. Science 290:521-523

Callaway RM, Ridenour WM (2004) Novel weapons: invasive success and the evolution of increased competitive ability. Front Ecol Environ 2:436-443. https://doi.org/10.1890/15409295(2004)002\%5b0436:NWISAT\%5d2.0.CO;2

Cappuccino N, Arnason JT (2006) Novel chemistry of invasive exotic plants. Biol Lett 2:189-193. https://doi.org/10.1098/ rsbl.2005.0433

Chen Z, Vertes A (2008) Early plume expansion in atmospheric pressure midinfrared laser ablation of water-rich targets. Phys Rev E 77:36316. https://doi.org/10.1103/PhysRevE.77.036316

Chen Z, Bogaerts A, Vertes A (2006) Phase explosion in atmospheric pressure infrared laser ablation from water-rich targets. Appl Phys Lett 89:41503. https://doi.org/10.1063/1.2243961

Cooks RG, Ouyang Z, Takats Z, Wiseman JM (2006) Detection technologies. Ambient mass spectrometry. Science 311:1566-1570. https://doi.org/10.1126/science.1119426

Gibb S, Strimmer K (2012) MALDIquant: a versatile R package for the analysis of mass spectrometry data. Bioinformatics 28:2270 2271. https://doi.org/10.1093/bioinformatics/bts447

Le Roux PC, McGeoch MA (2008) Rapid range expansion and community reorganization in response to warming. Glob Chang Biol 14:2950-2962. https://doi.org/10.1111/j.1365-2486.2008.01687.x

Macel M, de Vos RCH, Jansen JJ et al (2014) Novel chemistry of invasive plants: exotic species have more unique metabolomic profiles than native congeners. Ecol Evol 4:2777-2786. https:// doi.org/10.1002/ece 3.1132 
Marler MJ, Zabinski CA, Callaway RM (1999) Mycorrhizae indirectly enhance competitive effects of an invasive forb on a native bunchgrass. Ecology 80:1180-1186. https://doi.org/10.1890/00129658(1999)080\%5b1180:MIECEO\%5d2.0.CO;2

Nemes P, Vertes A (2007) Laser ablation electrospray ionization for atmospheric pressure, in vivo, and imaging mass spectrometry. Anal Chem 79:8098-8106. https://doi.org/10.1021/ac071181r

Petras D, Jarmusch AK, Dorrestein PC (2017) From single cells to our planet-recent advances in using mass spectrometry for spatially resolved metabolomics. Curr Opin Chem Biol 36:24-31

Schaffner U, Ridenour WM, Wolf VC et al (2011) Plant invasions, generalist herbivores, and novel defense weapons. Ecology 92:829-835

Senior JK, Potts BM, Davies NW et al (2016) Phylogeny explains variation in the root chemistry of eucalyptus species. J Chem Ecol 42:1086-1097. https://doi.org/10.1007/s10886-016-0750-7

Shrestha B, Vertes A (2009) In situ metabolic profiling of single cells by laser ablation electrospray ionization mass spectrometry. Anal Chem 81:8265-8271. https://doi.org/10.1021/ac901525g

Stinson KA, Campbell SA, Powell JR et al (2006) Invasive plant suppresses the growth of native tree seedlings by disrupting belowground mutualisms. PLoS Biol 4:e140. https://doi.org/10.1371/ journal.pbio. 0040140
Stopka SA, Agtuca BJ, Koppenaal DW et al (2017) Laser-ablation electrospray ionization mass spectrometry with ion mobility separation reveals metabolites in the symbiotic interactions of soybean roots and rhizobia. Plant J 91:340-354. https://doi.org/10.1111/ tpj. 13569

Walther G-R, Post E, Convey P et al (2002) Ecological responses to recent climate change. Nature 416:389-395. https://doi. org/10.1038/416389a

Wilschut RA, Silva JCP, Garbeva P, van der Putten WH (2017) Belowground plant-herbivore interactions vary among climatedriven range-expanding plant species with different degrees of novel chemistry. Front Plant Sci 8:1861. https://doi.org/10.3389/ fpls.2017.01861

Wu C, Dill AL, Eberlin LS et al (2013) Mass spectrometry imaging under ambient conditions. Mass Spectrom Rev 32:218-243. https ://doi.org/10.1002/mas.21360

Xia J, Sinelnikov IV, Han B, Wishart DS (2015) Metaboanalyst 3.0making metabolomics more meaningful. Nucleic Acids Res 43:W251-W257. https://doi.org/10.1093/nar/gkv380 